\title{
Du corps au cancer : la construction métaphorique de l'ennemi intérieur dans le discours militaire pendant la Guerre Froide. Partie 2
}

\section{Gabriel Périès}

\section{(2) OpenEdition \\ Journals}

\section{Édition électronique}

URL : http://journals.openedition.org/conflits/866

DOI : $10.4000 /$ conflits.866

ISSN : 1777-5345

Éditeur :

CCLS - Centre d'études sur les conflits lilberté et sécurité, L'Harmattan

Édition imprimée

Date de publication : 1 septembre 2001

ISBN : 2-7475-1118-9

ISSN : $1157-996 \mathrm{X}$

\section{Référence électronique}

Gabriel Périès, « Du corps au cancer : la construction métaphorique de l'ennemi intérieur dans le discours militaire pendant la Guerre Froide. Partie $2 »$, Cultures \& Conflits [En ligne], 43 | automne 2001, mis en ligne le 28 février 2003, consulté le 30 mars 2021. URL : http://journals.openedition.org/ conflits/866 ; DOI : https://doi.org/10.4000/conflits.866

Ce document a été généré automatiquement le 30 mars 2021.

Creative Commons License 


\title{
Du corps au cancer : la construction métaphorique de l'ennemi intérieur dans le discours militaire pendant la Guerre Froide. Partie 2
}

\author{
Gabriel Périès
}

L'Appareil de l'organisation ennemie comme corps

On placera en regard de cette corporéité du corps militaire qui associe « organiquement » un officier spécialisé avec un organisme particulier, la construction métaphorique, en quelque sorte, inverse : celle de l'organisation ennemie telle que la propose le général Diaz de Villegas dans son ouvrage intitulé, La guerra revolucionaria ${ }^{1}$ et quipermet l'assimilation de cette organisation à un corps. Ce texte espagnol de 1959 reprend l'ensemble de la casuistique de l'ESG en la matière et ses principaux lieux communs en les appliquant à la guerre civile espagnole. Toutefois, ce qui constitue l'intérêt majeur de cet ouvrage est qu'il est abondamment illustré de multiples représentations de l'ennemi et de son organisation ${ }^{3}$. Celles-ci sont tout à fait remarquables, car elles sont la plupart du temps des expressions métaphoriques qui renvoient aux cours mêmes de l'ESG, aux articles doctrinaux marquants de la DGR, qu'ils reprennent ou prolongent.

Figure 2 : illustration extraite deLa guerra revolucionariadu général Diaz de Villegas et son texte de légende.

\section{Texte $\mathrm{n}^{\circ} 3$}

«La Révolution est bien trop complexe pour qu'elle puisse émerger d'elle-même. Elle n'est jamais spontanée. Elle est préparée, elle est organisée, et elle est finalement déclenchée. Elle procède d'une technique. C'est au moment exact où elle éclatera que l"Appareil'se mettra à fonctionner. Personne ne se sera rendu compte de son organisation. C'est pour cela sans doute qu'il existe la possibilité de bloquer son processus de développement sans trop de souffrance. La Révolution, comme le disait Lénine et le recommandait Mao, doit toujours surgir dans les villes. C'est son milieu 
propice. Elle irradiera d'autant plus fort que la ville sera plus importante, la victoire alors n'en sera que plus concluante. La Révolution, cependant, n'oublie jamais le milieu rural dans lequel elle cherchera à s'enraciner par la suite $»^{4}$.

L'argument central du général Villegas repose sur le caractère non spontané de la Révolution. Celle-ci est obligatoirement le fruit d'un complot, de l'action d'un «Appareil» (Aparato en espagnol) . D'un point de vue lexical, on peut déterminer que c'est la charge sémantique de ce terme qui sert de socle à l'argumentation. Il se trouve être un des éléments constitutifs de la métaphore in praesentia, avec l'image de la page qui l'accompagne (voir figure $n^{\circ} 2$ ). Le mot structure dans l'ordre de la lecture la représentation et en fixe la compréhension. Le terme Appareil figure en plus dans l'image en tant que légende, instaurant la relation entre $E l$ aparato de la revoluciónet "Aparato »du texte qui, on le remarquera, se détache quelque peu de l'illustration par le biais des guillemets et de l'écriture italique ce qui renforce l'autonomie de la métaphore corporelle instituée par le dessin. Mise en relief, cette métaphore sert de médium entre les différents éléments désignant l'organisation communiste et le corps stylisé qui en représente l'unité organique.

A regarder de plus près la représentation produite par le général Villegas, on remarquera qu'au-dessus de la tête du personnage chargé de signifier le commandement général [mando general] se situe le symbole de l'Union Soviétique - la faucille et le marteau étoilés - et que ce qui est censé signifier le cerveau, el Estado Mayor[l'EtatMajor] est représenté par deux personnages discutant autour d'un bureau.

Dans tous les cas, on constate que le texte des cartouches et les illustrations latérales qui les accompagnent désignent non pas des organes mais des fonctions - mais, sur le plan doxique, la fonction ne crée-t-elle pas l'organe ? C'est en partie là que se trouve l'autre versant de la légitimité de la représentation organiciste de l'appareil révolutionnaire sous la forme d'un corps. Ce ne sont pas, effectivement, à proprement parler, des organes qui sont métaphoriquement désignés ici. Ce sont plutôt des fonctions hiérarchiquement coordonnées. La métaphorisation ne fait que leur donner la cohérence rhétorique globale. C'est, en fait, une hiérarchie qui est ainsi désignée.

$\mathrm{Au}$ niveau $\mathrm{du}$ cou et $\mathrm{du}$ torse $\mathrm{du}$ corps-appareilon observe le mando regional[Commandement régional]. Le mando provincial en capitales [Commandement provincial dans les capitales]est situé, quant à lui, au niveau de l'abdomen. Si des flèches de correspondance partent vers d'autres métaphores, les deux premières signalent deux cartouches placées juste sous les bras du personnage, ce qui accrédite l'analogie entre ses organes de préemption et les images qui désignent du côté droit, des instruments logistiques de movilización, golpes de mano, garages y tiendas de viveres[mobilisation, coup de main(sic), garages et dépôts de vivres] et du côté gauche, la disposition de armas previamente ocultas[d'armes préalablement cachées]. Toujours de l'abdomen sort ensuite ce qui désigne métaphoriquement des actions de sabotage, acción por sectores y celulas, sabotages. Ce sont des éléments lexicaux désignant des " organes" spécifiques du Parti Communiste qui sont utilisés ici : secteurs et celulas [cellules] -désignation tout aussi organiciste, du moins pour ce qui concerne le sens de ce dernier mot. Le dernier « organe » de l'appareil à sortir de l'abdomen est celui des servicios de información y enlace[services d'information et de liaison]. Enfin, les jambes sont signifiées par « las ordenes a las bases, formación de ejercito subversivo, acción celulas de base capitaneadas por un 'activista' ». [les ordres vers les "bases", la formation d'une armée subversive, l'action des cellules de base commandées par un « activiste »]. 
Si c'est bien une hiérarchie qui est signifiée, nous pouvons nous rendre compte que ce sont finalement des fonctions militaires qui sont désignées dans les différents cartouches. Cet ensemble fonctionnel à vocation guerrière se trouve référencé par un lexique lui aussi militaire : Mando general, Estado Mayor, mando regional, mando provincial, movilización, golpe de mano, sectores, sabotage, armas, servicios des información y enlace, ordenes, capitaneadas[por]. Les mots et syntagmes qui trouvent leurs reférenciation dans le lexique militaire orientent la métaphore de la Révolutionvers une compréhension spécifique propre au professionnel de la violence légitime. Celui-ci situe cette métaphore dans la logique de la guerre et de son organisation, donc relevant de son domaine de compétence.

Une remarque particulière doit être faite à propos de l'emploi de ce type de métaphore relayé par le recours à un lexique militaire. On peut en dégager une sorte de constante par rapport aux exemples précédents. Il nous apparaît que le recours à la métaphore organiciste de l'appareil, mise en relation avec la Révolution,réalise, en fait, l'établissement de l'analogie entre la représentation de la Révolution - Révolution qui ne peut être autre chose qu'une situation préalablement organisée - et une structure militaire. D'un côté, cela veut dire que pour analyser un processus social, le militaire n'a d'autre système de représentation et de compréhension référencée sur le plan organisationnel que celui auquel il participe professionnellement et qu'il projette comme modèle social ; de l'autre, que ce type de métaphorisation permet de désigner l'ennemi, et surtout de visualiser ce qui est défini comme clandestin et caché, à savoir les principaux « organes » de l'organisation révolutionnaire intimement associée à la Révolution elle-même : résumée au rôle d'un appareil clandestin, celle-ci se trouve de la sorte « incarnée».

Tératologie discursive pendant la guerre froide : entre chimères sociales et contrerévolution

Si on poursuit nos investigations sur l'unité de la représentation organisciste avec la MMC, il convient d'aborder maintenant le lien qui unit leur emploi à l'élaboration d'un discours politique, idéologique. A travers l'analyse du discours des cours à l'ESG que donne J. Monnerot ${ }^{5}$, un civil, on aura l'occasion de relever la prégnance de ce discours qui dresse une véritable tératologie discursive en créant de véritable chimères sociales à but rhétorique ; puis dans une seconde partie, on s'intéressera à l'emploi de la MMC et au discours organiciste dans le discours militaire de la contre-révolution.

Discours civil, métaphores organicistes et chimères sociales

«Les idées sont, de plus, contagieuses. Il y a des principes qui président aux contagions d'idées et aux épidémies psychologiques. On pourrait théoriquement déterminer la classe ou catégorie de ceux qui risquent, du fait des conditions objectives de leur biographie, d'y être sensibles, et il est théoriquement possible, mais ce n'est pour le moment qu'une vue de l'esprit, de les identifier et de tenter de les 'vacciner'à temps. Nous nous bornerons à signaler ici cet aspect théorique de la question. Notre objet est plus limité.

Donc l'intelligentsia russe s'était emparée du marxisme, avait recueilliprécieusement du socialiste allemand Kautsky cette opinion que les ouvriers ont besoin qu'on pense pour eux, s'était installée dans cette fonction de 'cerveau du prolétariat' : Le reste du corps doit obéissance au cerveau. Mais la volonté de puissance des membres les plus doués de cette intelligentsia, qui pour ainsi parler, se militarise pour vaincre, accommode plus ou moins consciemment le marxisme à ses propres besoins, l'infléchit $»^{6}$. 
La militarisation du discours civil s'effectue par le biais de l'établissement de métaphores organicistes et médicales. L'extrait du texte de la conférence du locuteur civil en atteste.

Formellement, cet extrait du cours qui analyse l'avancée de la révolution bolchevique en Russie, se décompose clairement en deux paragraphes articulés autour de la charnière donc. Celle-ci détermine la nature logico-argumentative de l'énoncé dans l'ordre de la consécution et sépare en deux temps le schéma discursif du locuteur qui peuvent être ainsi établis : dans le premier, l'auteur prétend aborder un aspect théorique de la circulation des idéologies et des doctrines politiques - révolutionnaires ; le second apparaît plus comme une explicitation historisante de ce qui a été avancé précédemment, l'Histoire donnant une exemplarité pratique aux affirmations théoriques. Bref, l'organisation de l'argumentation s'enclenche sur le schéma classique théorie-pratique.

Dans ce contexte argumentatif, la dimension tropologique du discours de J. Monnerot repose sur trois réseaux spécifiques qui convergent vers une militarisation des représentations du politique. Deux sont de nature métaphorique et analogique, tandis que le troisième relève plus de la connotation politique que du trope proprement dit, mais est intimement liée, comme nous allons le voir, aux deux autres. Articulées à la thématique de la pathologie infectieuse, ces procédures rhétoriques vont articuler des représentations organicistes de l'idéologie et de la société à une représentation technicienne de l'action politique fortement inspirée par les pratiques bolcheviques.

Le domaine de l'idéologie comme lieu d'apparition de pathologies infectieuses

Le premier de ces réseaux établit une relation métaphorique directe entre le domaine de l'idéologie et celui de la pathologie infectieuse. Ce qui relève des idées est automatiquement lié à l'adjectif contagieuses, au substantif contagionset à celui d'épidémie. J. Monnerot assure la pertinence de son discours en associant ces termes avec psychologique. Cet adjectif ajuste le lien énonciatif et ambigu entre la pathologie clinique - élargie au domaine quasi-psychiatrique - et la dimension béhavioriste des sciences sociales qu'il recouvre également. Le locuteur peut ainsi joindre, sous une forme de légitimité scientiste, ses métaphores aux termes de classes et de catégoriessociales, devenues sensibles à cette forme idéologico-pathologique de contagion : la propagande. On soulignera la dimension quasi policière de l'affirmation selon laquelle des conditions objectives de leur biographie permettraient de déterminer le degré de sensibilité à l'idéologie. Il s'agit bien de «cibler» de la sorte la dimension sociale d'une enquête individuelle qui peut également devenir un moyen de classification politique d'une population sur la base de critères sociaux.

Psychologique exprime alors l'utilité, puisqu'il est possible de méthodologiser le rapport entre la pathologie idéologique et les groupes sociaux. Le locuteur peut désigner à ses auditeurs les actions à mener. Les verbes à l'infinitif sont à cet égard particulièrement signifiants : tel un épidémiologue chargé de la prévention d'une maladie donnée au sein d'une population, J. Monnerot propose de déterminer la classe ou la catégorie, afin de les identifier clairement pour pouvoir les "vacciner». La connotation destructrice de ce dernier mot peut être envisagée du fait des guillemets, qui, s'ils en euphémisent le sens strict, soulignent de facto la métaphorisation médicale de l'élimination d'un germe, c'est-à-dire de l'idéologie incriminée.

A travers les verbes déterminer / identifier / « vacciner »qui expriment l'orientation de l'action - la fonction de l'infinitif résidant pour une large part dans l'expression de 
l'injonction ou de la forte recommandation - l'énonciateur rappelle que son enseignement a une fonction pratique. J. Monnerot ne se contente pas uniquement de désigner au professionnel de la violence ce qu'est l'ennemi, mais aussi comment le traiter, comment le détruire.

La représentation organique de la société, substrat métaphorique de la pathologie

Le second réseau métaphorique est celui qui, en interpénétration avec le premier, fait émerger une représentation organique de la société. Si, comme nous l'avons vu, le substrat dans lequel évolue la pathologie, est constitué de classeset de catégories, le locuteur en appelle à l'histoire de la révolution russe, pour y placer des mots qui appartiennent, directement ou allusivement, à son registre lexical : Intelligentsia russe et prolétariat fixent dès lors le contexte discursif : le locuteur parle de la révolution russe. Par leur appartenance au registre politique et idéologiquement marqué, ces deux expressions figent ce qui relevait, in abstentia, de la métaphore généralisante : idées et épidémies pathologiques, ont maintenant, pour référent, in praesentia, marxisme, terme désignant la seule idéologie dont le locuteur parle.

Ce sont les composantes sociales responsables de la corruption-infection, de l'apparition de la pathologie, qui subissent la métaphorisation organiciste. Intelligentsia se mue en cerveau du prolétariat et le prolétariat en reste du corps. Mais ce corps possède une tête monstrueuse : c'est de l'intelligentsia d'où sortent les membres, terme signifiant tout à la fois l'appartenance à un groupe et qualifiant des organes de préemption ou de locomotion. Or, ces membres sont animés d'une volonté de puissance : lorsque les clercs trahissent, des chimères sociales apparaissent.

Un autre corps cependant peut faire contrepoids face à cette aberration. Il est sémantiquement induit par une sorte d'argumentation allusive a contrario. Il transparaît à travers la licéité de la dimension politico-militaire du discours de J. Monnerot.

Discours connotatif et bolchévisation de l'action politique

Le troisième réseau est celui que lui confère son sens politico-militaire et autoritaire. Contrairement aux deux autres, il n'est pas, à strictement parler, métaphorique mais connotatif. Il provient de la déqualification morale du politique induite par les métaphorisations médicales et organicistes et la valorisation indirecte du militaire, de son mode d'action érigé en technique efficace.

Les aspects autoritaires du discours de J. Monnerot sont en rapport étroit avec une gradation dans la progression de ses propositions théoriques, faites évidemment, pour ne pas le rester. Pour commencer, d'emblée, il prend, en tant qu'intellectuel, la responsabilité majeure de désigner l'ennemi aux professionnels de la force. Il place, pour ce faire, l'ensemble des déterminants sociaux qu'il étudie au centre de la relation ami-ennemi, c'est à dire dans le terrain de la coaction ${ }^{7}$-coercition-annihilation.

Un autre point situe la dimension autoritaire du contenu de cette conférence. Entre intelligentsia et prolétariat, cerveau et corps, la seule relation socio-politique existante et explicitée par J. Monnerot, est le rapport hiérarchique d'obéissance organique, donc naturelle ${ }^{8}$.

Ceci dit, l'analyse du discours de J. Monnerot aux officiers stagiaires de l'ESG présente un infléchissement. Le locuteur induit une sorte de déplacement-exaltation de certaines valeurs, qui, dans son phrasé, ne sont pas négativement connotées, bien au contraire. Ce déplacement du négatif vers le positif concerne la thématique de la 
victoire politique réalisée sur le mode militariste et un rappel à une vulgate nietzschéenne : la volonté de puissance des membres les plus doués(...) se militarise pour vaincre. Entre l'obéissance sociale et la victoire, il faut, pour asseoir l'autorité des membres les plus vigoureux de l'intelligentsia, passer par le domaine du militaire et tordre alors les principes de base qui légitiment le combat politique démocratique ou les idéaux de transformation sociale.

Car, ce qui importe aux yeux du locuteur, c'est la dimension instrumentale de l'idéologie : pour atteindre le pouvoir, il faut s'en accommoder, l'essentiel étant, pour assurer ses propres besoins, del'infléchir. Les croyances ne sont pas faites pour les maîtres, dont le seul moteur de l'action est la volonté de puissance. Si le locuteur reconnaît l'existence d'une idéologie possédant un substrat social, c'est afin que l'« élite » l'utilise pour prendre ou conforter son pouvoir ; la seule relation sociale réelle et efficace étant, dans ce cadre, la relation d'autorité.

Ainsi, pour queles plus doués prennent le pouvoir, pour qu'ils nouent leur destin avec la victoire sociale, ils doivent militaris[er]leur volonté. Et l'on ne peut s'empêcher de penser au léninisme qui, en accommod[ant] plus ou moins consciemment, [une idéologie] le marxisme à ses propres besoins(...) l'a transformé en doctrine de prise du pouvoir par la constitution d'un parti militarisé. En d'autres termes, J. Monnerot vient de proposer, ici de façon indirecte, comme modèle d'action psychologique, les techniques du bolchevisme tout en disqualifiant, derrière un nietzchéisme de façade, toute idéologie de transformation sociale. Seules les techniques d'action doivent être radicales. La chimère sociale constituée des métaphores organicistes a été rhétoriquement utile ; si, dans un premier temps, elle a permis la production d'un être imaginaire monstrueux, d'une représentation de l'utopie léniniste, ce repoussoir idéologique permet dans un second temps de rejeter ce qui est idéologique au profit d'une captation légitime des techniques militaristes du léninisme alors neutralisées.

Contre-révolution, métaphore médicale et décontextualisation

C'est peu de temps après la parution du texte Guerre révolutionnaire et médecine moderne que nous avons analysé plus haut, que se tient à l'ESG un débat. Des officiers, stagiaires en 2ème année organisent le 18 juin 1957, date symbolique s'il en est, une table ronde intitulée «La guerre révolutionnaire $»^{9}$ en trois temps : I. Tactiques révolutionnaires et contre-révolutionnaires; II. Nécessité et conditions d'une stratégie contrerévolutionnaire; III. Questions posées à la suite du débat. Et ils vont filer la métaphore jusqu'à produire un phénomène de décontextualisation de l'énoncé pour aboutir finalement à un complet retournement de la DGR contre l'autorité civilo-politique.<// r2r:section1>

Ce discours propose un argumentaire dans un contexte polémique. En 1957, la bataille d'Alger fait rage et ce dont les différents intervenants vont en fin de compte débattre c'est de la légitimité de la pratique des interrogatoires "policiers ", de la sortie de la bataille en cours, et surtout, de la détermination du contenu politique qu'il convient de lui attribuer.

Pour faire l'analyse de ce document en mettant l'accent sur l'emploi de la MMC, on repérera le phénomène dans le débat du 18 juin 1957. Ce qui nous amènera à relever le lien entre l'emploi de ce type de métaphore filée et la décontextualisation de certaines pratiques; on pourra, dès lors, préciser comment l'emploi de la MMC contribue au retournement et à l'amplification rhétorique d'un rôle interventionniste-médicinal de l'Armée relatif à l'Algérie sur le territoire national. On observera alors comment la 
MMC, appuyée sur des représentations organicistes, sert de socle argumentatif à l'élaboration de pratiques professionnelles qualifiées de contre-révolutionnaires.

[a] «Si l'action est très importante pour préserver une population encore saine du virus révolutionnaire, elle n'obtient que des résultats décevants quand elle est employée sur une population déjà contaminée $»^{10}$.

$[\mathrm{b}]$ « La passion du sport les immunisaitcontre le communisme $»^{11}$.

[c] «Les communistes bénéficient de l'aide consciente ou inconsciente de tous ceux qu'ils ont réussi à intoxiquer : neutralistes, progressistes (...) $»^{12}$.

[d] «Si nous savons conduire efficacement l'éducation civique et l'action psychologique dans nos forces Armées, cette action atteindra par 'capillarité l'ensemble de la Nation $»^{13}$.

[e] « II- NECESSITE ET CONDITION D'UNE STRATEGIE CONTRE-REVOLUTIONNAIRE

Je vous arrête là sur une pente dangereuse : si j'ai bien compris ce que vous venez de nous expliquer, l'action adverse consiste à s'infiltrer dans la société, d'abord à la manière d'un virus qui pénètre les esprits, puis en pénétrant le corps, en noyautant de l'intérieur toutes les cellules dont l'assemblage constitue l'architecture sociale et politique du pays; enfin seulement se durcissant dans une sorte de cristallisation militaire, à faire éclater toutes les structures devenues creuses.

Le remède que vous proposez c'est en somme de prévenir l'adversaire en durcissant nous-mêmes à temps nos structures, en les 'militarisant' (...) là je dis « attention »! Car il ne faut pas qu'il se contente d'arrêter provisoirement la maladie $»^{14}$.

[f] « Pensons bien les caractéristiques de cette thérapeutique :

- D'abord vous êtes le premier à reconnaître que le traitement ne peut s'appliquer seulement à la partie malade. C'est le corps entier qu'il faut mettre en état de défense. Nous savons tous que l'Indochine a été perdue à Paris, que la maladie algérienne est une maladie française.

- Ensuite, cette phase 'pré-insurrectionnelle' dont vous avez parlé est extrêmement longue. A vrai dire, elle n'a pas de limites! S'il faut militariser une société pour la mettre en état de défense contre la subversion idéologique, nous devons dire que nous devons vivre indéfiniment en société militarisée.

- Le problème est bien en effet de 'durcir' nos structures sociales et politiques pour rendre imperméable à l'action adverse la Masse organisée que constitue le corps de la Nation. Le "durcissement » doit être général, mais nuancé en ses parties selon le degré de la maladie $»^{15}$.

$[\mathrm{g}]$ « III- QUESTIONS POSEES A LA SUITE DU DEBAT

Il y a toujours des 'contradictions internes' dans une société (...) mais s'il existe une véritable organisation révolutionnaire qui s'en sert, il est illusoire et même dangereux de prétendre mettre fin à la lutte en les faisant disparaître. On ne soigne pas une maladie infectieuse en traitant les plaies apparentes du malade; il faut tuer le microbe, et ensuite cicatriser les plaies (ceci dans l'ordre de priorité, pas obligatoirement dans le temps) ${ }^{16}$.

Métaphores filées et décontextualisation 
Nous savons que l'emploi de la MMC que nous avons également rencontré lors des analyses des doctrines anglo-saxonnes de la contre-guérilla dans les années 1955 concourt, dans le cadre de la "guerre révolutionnaire", à placer le soldat dans une attitude interventionniste au sein de la société représentée comme un véritable corps souffrant, couvert de plaies, voire, unmalade. Or on remarquera que le texte de la conférence prolonge le texte "Médecine moderne et guerre révolutionnaire». S'il décrit métaphoriquement la pacification dans le cadre algérien, le syntagme médecin des sociétés[cf. Annexe] a bel et bien une vocation généraliste, si c'est bien de la société algérienne dont il est question... Les interlocuteurs de l'ESG filent dès lors eux-mêmes cette métaphore, et sont prêts à situer l'origine des maux dont souffre l'Empire en métropole, car l'ensemble des métaphores relevées constitue en fait une représentation de la pathologie dont souffrirait la France : la maladie algérienne est une maladie française [f], ce qui implique une intervention des spécialistes, des confrères spécialistes en France [cf. Annexe a]. En d'autres termes, la problématique de la "guerre révolutionnaire ", si l'on suit la métaphore entre mai et juin 1957, s'est transférée de l'Algérie au territoire national; à la seule différence que, cette fois-ci, il ne s'agit pas d'éliminer un agent pathogène ayant pour référent direct le FLN ou l'ALN, mais bien de supprimer le Parti Communiste, le virus, ou tout autre microbe [g]intoxiquant la France.

On notera que c'est le vocabulaire et les expressions de l'ennemi qui sont à leur tour métaphorisés. L'expression "contradictions internes", véritable exhibition de la captation du discours marxiste-léniniste, trouve sa place dans la construction métaphorique du corps de la nation : ce sont desplaies qui doivent être cicatrisées par le médecin. Le termemédecin n'apparaît pas, ce n'est pas nécessaire puisque c'est le locuteur lui-même devant son public qui en assume le rôle, qui en fait un vecteur rhétorique de son acte d'autorité dans un contexte général prégnant: celui de l'explicitation de la DGR.

Métaphore médico-chirurgicale : retournement et amplification

C'est dans ce contexte déplacé que se déploie la notion de contre-révolution. Dans sa dimension métaphorique ${ }^{17}$, elle instaure un véritable retournement que réalise, toujours sur la base de l'emploi de la MMC, un des locuteurs.

Premièrement, il apparaît que ce n'est pas la méthode de l'ennemi qui est en cause : le locuteur n'a aucun problème à exempter les aspects techniques de toute condamnation morale car, comme il le rappelle dans le dialogue-débat: " nous pensons qu'il ne faut pas confondre les causes de la guerre révolutionnaire et ses procédés ${ }^{18}$. Cette séparation entre la technique et les préceptes idéologiques ou moraux qui la mettent en œuvre s'exprime à travers l'aisance avec laquelle le locuteur dépolarise, puis inverse les référents dans l'emploi des métaphores, en particulier celles qui touchent le domaine organicisteorganisationnel : elles sont extraites de la description de l'action de l'ennemi pour être basculées sur celle de l'ami ; l'objet de l'action devient l'organisation du corps de la nation, désigné comme Masse organisée, qu'il s'agit d'encadrer, de diriger dans un ensemble militarisé de structures porteuses d'un civisme revigoré.

D'un autre côté, lorsqu'il s'agit d'apprécier lesnécessité et condition d'une stratégie contrerévolutionnaire, un des locuteurs inverse également l'emploi tropologique de la MMC pour revenir au sens premier des référents ; en effectuant cette opération, il en réalise une seconde, tout aussi rhétorique. Partant, semble-t-il, d'un postulat critique, un des locuteurs relève le risque de militarisation permanente de la société [e]; toutefois, le contenu de la phrase alarmiste ne débouche pas sur une critique de l'énoncé de son 
interlocuteur ; bien au contraire, il prend à contre-pied son propre phrasé ; il aboutit ainsi à une radicalisation: le attention !devient une véritable mise en garde : la contrerévolution exige l'impérieuse nécessité de résultats réels et définitifs. Elle refuse que l'élimination de l'agent infectieux ne soit que transitoire, que le durcissement que l'on croyait, au prime abord, dangereux pour la société, ne se content[e]que d'arrêter provisoirement la maladie [e].

$\mathrm{Au}$ terme de ce travail, nous pouvons considérer que l'emploi de la MMC dans le discours militaire pendant la Guerre Froide est pertinent. Il repose sur deux présupposés essentiels : la rémanence des représentations organicistes relatives à tout ensemble organisé et hiérarchisé d'un côté, et de l'autre: la neutralité de l'acte scientifique et empirique corrélé à la dimension moralement positive de la médecine. Or, cette neutralité doxique n'est pas anodine: elle permet la construction d'une représentation spécifique de l'ennemi qui légitime, sur la base des complémentarités discursives et des analogies, l'action sociale et, plus largement, politique. En effet, la conclusion des énoncés qui intègrent la MMC recèle, presque toujours, un gommage rhétorique de la séparation institutionnelle des rôles et des fonctions entre le civil et le militaire. La revendication $\mathrm{du}$ rôle de médecin par le militaire fait ainsi symboliquement fusionner les deux domaines de compétence où l'un est normalement soumis au premier: dans l'ordre médical général, c'est le médecin qui établit le diagnostic tandis que le chirurgien opère en fonction de ce diagnostic. Dans ce cadre énonciatif, la représentation discursive de l'ennemi devient essentielle; sa dénomination métaphorique spécifique intègre le chaînage logico-argumentatif luimême dans le jeu des représentations organicistes: celles-ci facilitent alors la construction idéale de structures de décision fusionnées entre les mains du professionnel de la violence légitime; mais celle-ci l'est-elle encore? Nous pensons, effectivement, que cette dénomination particulière de l'ennemi comme de l'ami, qui fait du corps social un enjeu guerrier, exprimein fine une autonomisation croissante du secteur militaire par rapport au politique et en signale la crise à venir.

Annexe

a) «Le praticien est-il impuissant devant un cas qui déroute son diagnostic et met en échec sa thérapeutique ? Il appelle en consultation des confrères spécialisés. Il établit un bilan physiologique à l'aide de radiographies diverses, de mesures électriques, d'analyses et de dosages minutieux.

Puis, l'état général du patient exactement apprécié et l'affection rebelle cernée, il s'efforce - avec des hormones ou des vitamines - de déclencher, d'entretenir et de renforcer la réaction organique authentique (...) Des démarches analogues ne peuventelles être recommandées à notre médecin des sociétés encore attardé aux seuls antibiotiques? (Anonyme, Guerre révolutionnaire..., op. cit, pp.49-50).

b) «Sur ce terrain biologiquement actif, secrétant lui-même ses anticorps, les agents spécifiques extérieurs pourront agir avec une efficacité renforcée, 'suractivée' par l'auto-défense de la santé. L'armée - dans cette thérapeutique - n'est pas lancée immédiatement dans l'action et elle n'entreprend pas d'assumer, seule, toutes les tâches. Quand les 'hormones', les 'vitamines' du corps social ont renouvelé l'ambiance générale (...) l'armée - agent spécifique extérieur et non hormone - peut agir. Brutalement s'il le faut, mais avec l'assentiment, l'appui, l'encouragement de toutes les cellules, elle frappe les éléments réfractaires à l'ordre de la santé; elle est prête à éliminer physiquement - dans une 'lyse' définitive - les facteurs pernicieux qui 
n'auraient pas été 'phagocytés' auparavant » (Anonyme, Guerre révolutionnaire..., op. cit., p.51).

Retour au début

\section{NOTES}

1. . Général Diaz de Villegas, La guerra revolucionaria. La técnica de la revolución y la acción psicológica, el arma secreta del marxismo, Ediciones Europa, Madrid, España, 1959. L'ouvrage est préfacé par Luis Carrero Blanco, à l'époque « dauphin » de Franco. Ce texte a, par ailleurs, été trouvé en Argentine lors de nos recherches.

2. Sur ce plan, la bibliographie présentée par le général Villegas reprend les principaux documents alors en circulation en France sur ce thème.

3. . Dans les travaux des stagiaires étrangers, on relèvera dans ceux du lieutenantcolonel Rougueris, de l'Armée grecque : « En somme l'AFTOAMINA (autodéfense organisée par les communistes grecs en 1945-1947) est le cœur qui fait circuler le sang dispensateur de vie ; les guérillas sont le corps agissant sur ordre du cerveau, lequel cerveau est 'le Parti' ». Voir, Commission n 5, La guerre idéologique dans le monde, ESG, 68ème promo, 1er cycle, 1954-1955 1ère partie, Aspect général de la guerre idéologique en Grèce, pp. 2-3.

4. . Ibid., p. 94.

5. . Pour connaître le locutur : J-M Heimonet, Jules Monnerot ou la démission critique, 1932-1990. Trajet d'un intellectuel vers le fascisme, Paris, Kimé, 1993.

6. . J. Monnerot, «L'action psychologique dans la guerre », ESG-Etudes générales 2ème Cycle, mai 1956, p. 6.

7. . Dictionnaire Le Robert : Coaction : n. f (XIII s. du lat. coactio , rec. cogere, connaître). Action de priver de la liberté de choix. (...).

8. . Pour les implications méthodologiques et représentationnelles de l'organicisme, voir : P. Birnbaum, La fin du politique, Paris, Seuil, 1975. En particulier, le chapitre IV intitulé « Organicisme, société de masse et idéologie », p. 87.

9. Débat entre les commandants Hogard, Cogniet, Renaudin, ESG, 2ème cycle, La guerre révolutionnaire, Tactiques révolutionnaires et contre-révolutionnaires, 18 juin 1957.

10. . Débat entre les commandants Hogard, Cogniet, Renaudin, ESG, 2ème cycle, La guerre révolutionnaire, III Questions posées à la suite du débat, 18 juin 1957, p. 7. Dorénavant « Débat... ».

11. . Ibid., p. 10.

12. . Ibid., p. 2.

13. . Ibid., p. 6 .

14. . Ibid., pp. 6-7.

15. . Ibid., p. 7.

16. . Ibid., seconde partie, p. 2.

17. . Dans la culture contre-révolutionnaire, l'emploi de la métaphore organiciste et médico-chirurgicale est chose ancienne ; il suffit simplement de remonter au XIXème 
siècle pour le voir en situation comme dans cette chronique de l'écrasement de la Commune par les Versaillais : « Paris cependant revint à lui ; les barricades avaient été effacées du sol ; les traces du combat disparaissaient. (...) La France, 'noble blessée' retrouvait sa vitalité. La Commune avait été l'abcès qui avait failli causer sa perte ; nous avions crevé cet abcès à coups de bistouri, et cette guérison se déclarait ». A. Hans, Souvenirs d'un volontaire versaillais, Paris, E. Dentu Edit., 1873, p. 210.

18. . Débat..., op. cit., seconde partie, p. 2.

INDEX

Index chronologique : guerre froide

Mots-clés : contre-insurrection, Doctrine militaire, ennemi, Militaires, question coloniale, sécurité intérieure

Index géographique : Algérie 